\title{
Learning from experiences: quantitative and qualitative analysis of patient experiences during the inpatient admissions process Kathleen Sheehan* and Tom Burns
}

\author{
Address: University of Oxford, Department of Psychiatry, Warneford Hospital, Oxford, OX3 7JX, UK
}

* Corresponding author

from WPA Thematic Conference. Coercive Treatment in Psychiatry: A Comprehensive Review

Dresden, Germany. 6-8 June 2007

Published: 19 December 2007

BMC Psychiatry 2007, 7(SuppI I):S48 doi:10.1 I86/I47I-244X-7-SI-S48

This abstract is available from: http://www.biomedcentral.com/I47I-244X/7/SI/S48

(c) 2007 Sheehan and Burns; licensee BioMed Central Ltd.

\section{Objectives}

The treatment of patients against their will has long been a controversial issue in psychiatric care. Recent research has focused on whether patients perceive they have been coerced into treatment, regardless of whether objectively coercive measures were used. Using both quantitative and qualitative analysis, this study examined perceptions of coercion in a population of patients admitted to psychiatric hospital in the United Kingdom.

\section{Methods}

Patients admitted to five adult psychiatric wards were recruited to participate in an interview about their experiences of the hospitalization process. 164 patients were interviewed during the first week of their admission. 12 of these patients subsequently participated in an in-depth interview about their experiences of psychiatric hospitalization 12-24 months following the index admission.

\section{Results}

Of the 164 patients recruited to participate, 128 patients (78\%) were admitted voluntarily and 36 patients $(22 \%)$ were admitted involuntarily under a section of the Mental Health Act. Voluntarily admitted patients reported significantly lower levels of perceived coercion than involuntarily admitted patients $(2.4+/-1.7$ vs. $4.1+/-1.2 ; Z=-5.33$, $\mathrm{p}<0.001)$. Thematic analysis of the in-depth interviews highlighted the role of legal status, perceptions of choice and therapeutic relationship in shaping patient experiences during the hospitalization process. Further quantitative analyses will examine the association of sociodemographic, clinical and experiential variables with perceived coercion. Thematic analysis will systematically explore the experiences of patients in their own words.

\section{Conclusion}

By providing a better understanding of patients' experiences, this study may aid the development of policies and interventions for reducing negative experiences during psychiatric hospital admissions. 